\title{
A STUDY OF THE PRELOAD FORCE IN METAL-ELASTOMER TORSION SPRINGS
}

\author{
Wojciech SIKORA*, Krzysztof MICHALCZYK*, Tomasz MACHNIEWICZ* \\ "Faculty of Mechanical Engineering and Robotics, Department of Machine Design and Technology, \\ AGH University of Science and Technology, al. Mickiewicza 30, 30-059 Kraków, Poland \\ ${ }^{*}$ Faculty of Mechanical Engineering and Robotics, Department of Strength and Fatigue of Materials and Structures, \\ AGH University of Science and Technology, al. Mickiewicza 30, 30-059 Kraków, Poland \\ wosikora@agh.edu.pl, kmichal@agh.edu.pl, machniew@agh.edu.pl
}

received 10 June 2016, revised 29 November 2016, accepted 2 December 2016

\begin{abstract}
Neidhart type suspension units composed of metal-elastomer torsion springs can be a good alternative to steel helical springs in applications such as vibration absorbers or vehicle suspension systems. Assembling this type of spring requires initial preload of the elastomeric working elements, which determines their operating properties. The results of experimental tests and numerical simulations concerning the preload of elastomeric working elements in Neidhart type suspension units are presented in the paper. The performed research made it possible to propose a new calculation model for determining the preload force value acting on the elastomeric cylindrical elements applied in this type of suspension unit. The results obtained using the proposed model exhibit good convergence with FEM simulation results within the range of the tested geometrical and material properties.
\end{abstract}

Key words: Hyperelastic Materials, Neidhart Spring, Metal-Elastomer Spring

\section{INTRODUCTION}

Metal-elastomer elastic joints exhibit, as opposed to metal springs, good damping properties, which is the reason behind their wide application in vibration reduction systems or rotating machinery suspension systems, among others. Generally, this type of joint consists of rectangular shaped elastomeric elements bonded to rigid plates (Gent et al., 2007). However, such a construction has significant limitations, because, in the case of compression loading, nonuniform stress distribution in elastomeric elements causes a risk of extensive heat generation (Banić et al., 2012), stress concentration and, as a result, damage of the element. These phenomena are the reasons why, in the design practice for vibration isolators, deformations of compressionloaded elastomeric elements are limited to about $10-15 \%$ of the elastomeric component thickness (Rivin, 2003). Significantly preferable stress distributions can be obtained when an elastomeric element has a cylindrical shape and is loaded radially by two rigid plates (Neidhart R., 1969). Rivin and Lee demonstrated, by means of experiment (1994) and by numerical calculations (1996), that elastic joints of such a construction can withstand deformations in the compression mode even as high as $40 \%$, without the risk of the unfavorable phenomena described above. An example of the practical application of such a construction is the Lastosphere vibration isolator, produced for many years by the Lord Corporation.

It should be noted, however, that these structures were not able to compete with coil springs in terms of the maximum achievable deformation. Only the metal-elastomer torsion joint patented by Hermann Neidhart and its later modifications have made it possible to obtain deformations characteristic for helical springs, and damping properties characteristic for rubber-like materials.
The construction of a metal-elastomer torsion spring, called from the name of its inventor - a Neidhart spring, was patented in 1955 (Neidhart H., 1955) and was a development of structures applied earlier in vehicle suspension systems. Over the years, the number of applications of Neidhart springs has increased and the field of their utilization also include the examples outside the automotive industry. Currently, among the numerous examples of this solution's applications, supports of vibrating machinery (vibrating screens, vibrating conveyors), belt and chain tensioners, vibration isolators, pressure rollers and so on can be mentioned (Fig.1).

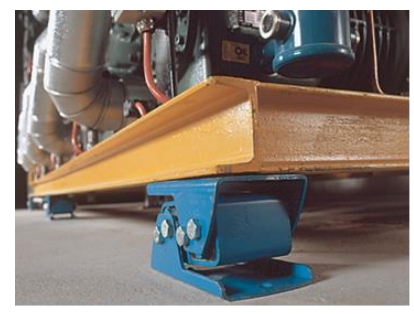

a)

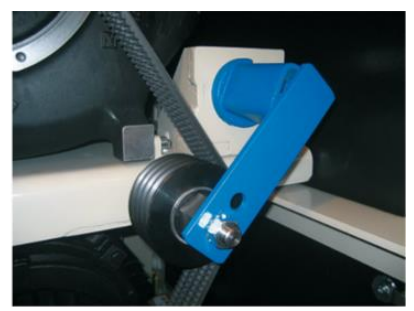

b)
Fig.1. Examples of typical applications of Neidhart springs: a) vibration isolation; b) transmission belt tensioner (http://www.rosta.ch)

Although Neidhart springs have been used for more than six decades, no extensive analysis of this construction can be found in the literature (Wodziński, 2003). The current situation is not conducive to the popularization of this solution, due to the lack of information concerning the rules for designing such springs and the influence of geometrical and material parameters on their operating properties. The static and dynamic properties of metalrubber torsion springs depend on their geometry and the composi- 
tion of the rubber compound (Samaca Martinez et al., 2013; Hassan et al., 2012; Chokanandsombat and Sirisinha, 2013) used to manufacture working cylinders. By making modifications in these two fields, one can obtain such spring properties that are most suitable in certain applications. Therefore, the article shows the results of experimental tests conducted in order to define the characteristics of rubber compounds used to produce the working elements of the tested springs. In the second part of the article, the relation between dimensions of the spring elements and the lateral force required to exert initial deformation of rubber cylinders is developed. There are, to the knowledge of the authors, no such formulas available in the literature that would allow one to approximate the relation between the geometric parameters of Neidhart type springs and assembly preload force. Knowledge of this force may be utilized in the design process of springs with specified reaction torque characteristics.

The aim of the study is to analyze the influence of the geometrical and material parameters of metal-elastomer torsion springs on the value of the preload reaction force between the rigid metal elements of the spring and elastomeric working elements.

\section{CONSTRUCTION OF NEIDHART SPRINGS}

A typical Neidhart type spring consists of an outer square tube, inner square shaft and four cylindrical elastomeric elements that are mounted between the tube and shaft in such a way that the shaft is rotated with respect to the tube by $45^{\circ}$, as shown in Fig. 2. It can be seen in Fig. $2 a$ that elastomeric cylinders are significantly compressed in a radial direction, which provides the initial preload of the spring.

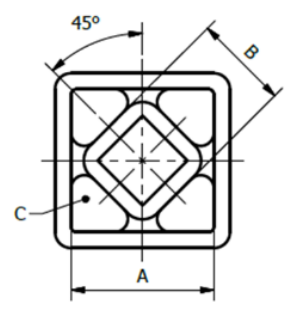

a)

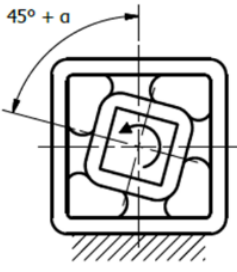

b)
Fig.2. Construction of the metal-elastomer spring: a) unloaded spring; b) loaded spring; A - inner dimension of the square tube, B - outer dimension of the shaft, $\mathrm{C}$ - elastomeric working element

As shown in Fig. 2b, the spring transfers torsional loads, however, it can also withstand lateral loads. The angular movement of the shaft with respect to the tube should be limited to only about $30^{\circ}$ due to the risk of unacceptably high deformation and strain of the elastomeric elements. The spring units shown in Fig. 2 can be mounted into sets as shown in Fig. 1a to transfer lateral movements.

\section{HYPERELASTIC MATERIAL MODEL}

Rubber, being an elastomer, is classified as a hyperelastic material. In the literature, one can find many models of such materials, of which the best known are the Neo-Hookean, Mooney-Rivlin, Arruda-Boyce and Ogden models (Bower, 2010; Boyce and Arruda, 2000; Kim et al., 2012). Besides the listed ones, due to significant differences in the properties of hyperelas- tic materials, there are many other models that have been developed for specific groups of materials (Lu et al., 2010).

Elastomeric material models are usually presented as a function of the elastic strain energy density. This is a starting point to determine the stress in a given state of load. To determine the parameters of the elastomer material, the static test of axial tension, compression or shear of an appropriately shaped test sample should be performed on a material testing machine. The chosen material model parameters can be obtained by fitting the theoretical curve to the experimental one.

The Mooney-Rivlin model (Mooney, 1940; Rivlin, 1948; Baranowski et al., 2012) is utilized in this paper. In this model, the strain energy density function $U$ can be expressed in the form (Bower, 2010):

$U\left(\overline{I_{1}}, \overline{I_{2}}\right)=C_{10} \cdot\left(\overline{I_{1}}-3\right)+C_{01} \cdot\left(\overline{I_{2}}-3\right)$

$+D_{1} \cdot(J-1)^{2}$

where: $C_{10}, C_{01}, D_{1}$ - material constants in $\mathrm{MPa}$.

$\overline{I_{1}}=J^{-2 / 3} \cdot I_{1}$

$\overline{I_{2}}=J^{-4 / 3} \cdot I_{2}$

where: $I_{1}, I_{2}$ - invariants of the left Cauchy-Green tensor B.

$\mathbf{B}=\mathbf{F} \cdot \mathbf{F}^{T}=\left[\begin{array}{ccc}\lambda_{1}{ }^{2} & 0 & 0 \\ 0 & \lambda_{2}{ }^{2} & 0 \\ 0 & 0 & \lambda_{3}{ }^{2}\end{array}\right]$

$I_{1}=\operatorname{tr}(\mathbf{B})=\lambda_{1}{ }^{2}+\lambda_{2}{ }^{2}+\lambda_{3}{ }^{2}$

$I_{2}=\frac{1}{2}\left[(\operatorname{tr} \boldsymbol{B})^{2}-\operatorname{tr}\left(\boldsymbol{B}^{2}\right)\right]=\lambda_{1}{ }^{2} \lambda_{2}{ }^{2}+\lambda_{2}{ }^{2} \lambda_{3}{ }^{2}+\lambda_{3}{ }^{2} \lambda_{1}{ }^{2}$

$\boldsymbol{F}=\left[\begin{array}{ccc}\lambda_{1} & 0 & 0 \\ 0 & \lambda_{2} & 0 \\ 0 & 0 & \lambda_{3}\end{array}\right]$

$J=\operatorname{det}(\boldsymbol{F})=\lambda_{1} \cdot \lambda_{2} \cdot \lambda_{3}$

where: $\mathbf{F}$ - deformation gradient tensor, $\lambda_{1}, \lambda_{2}, \lambda_{3}$ - principal stretches.

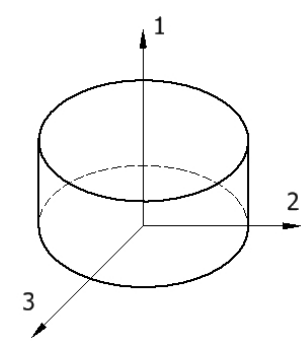

Fig. 3. Coordinate system for a cylindrical sample

Material testing of the rubber samples was based on an uniaxial compression test in direction 1 (Fig. 3). For such a case, it can be assumed that $\lambda_{2}=\lambda_{3}$ :

$\lambda_{2}=\lambda_{3}=\sqrt{\frac{J}{\lambda_{1}}}=\sqrt{\frac{J}{\lambda}}$

$\lambda_{1}=\lambda$

To simplify the material model, a specimen was treated as being made from an incompressible material thus $J=1$. This assumption is very close to reality in the case of rubber. True princi- 
pal stresses can be obtained from the formulas presented below (Bower, 2010):

$\widetilde{\sigma_{l j}}=2\left[\left(\frac{\partial U}{\partial I_{1}}+I_{1} \frac{\partial U}{\partial I_{2}}\right) B_{i j}-\left(I_{1} \frac{\partial U}{\partial I_{1}}+2 I_{2} \frac{\partial U}{\partial I_{2}}\right) \frac{\delta_{i j}}{3}\right.$

$\left.-\frac{\partial U}{\partial I_{2}} B_{i k} B_{k j}\right]+p \delta_{i j}$

$\widetilde{\sigma_{11}}=\frac{4}{3}\left[C_{10} \cdot\left(\lambda^{2}-\frac{1}{\lambda}\right)+C_{01} \cdot\left(\lambda-\frac{1}{\lambda^{2}}\right)\right]+p$

$\widetilde{\sigma_{22}}=\widetilde{\sigma_{33}}=-\frac{2}{3}\left[C_{10} \cdot\left(\lambda^{2}-\frac{1}{\lambda}\right)+C_{01} \cdot\left(\lambda-\frac{1}{\lambda^{2}}\right)\right]+p$

$p=\frac{\widetilde{\sigma_{11}}+\widetilde{\sigma_{22}}+\widetilde{\sigma_{33}}}{3}$

Because the specimen sidewall is unconstrained therefore $\widetilde{\sigma_{22}}=\widetilde{\sigma_{33}}=0$ and true stress in the direction of the compression axis is described by:

$\widetilde{\sigma_{11}}=2 C_{10} \cdot\left(\lambda^{2}-\frac{1}{\lambda}\right)+2 C_{01} \cdot\left(\lambda-\frac{1}{\lambda^{2}}\right)$

After dividing both sides of (2) by $\lambda$, the engineering stress was obtained:

$\sigma_{11}=2 C_{10} \cdot\left(\lambda-\frac{1}{\lambda^{2}}\right)+2 C_{01} \cdot\left(1-\frac{1}{\lambda^{3}}\right)$

$\lambda=1+\varepsilon$

$\varepsilon=\frac{z-z_{0}}{z_{0}}$

where (Fig. 4a): $\varepsilon$ - strain, $z_{0}$ - initial sample height, $z$ - sample height during test.

The relationship (3) for compressive stress on a central axis is necessary for determining the rubber material constants. Comparing equation (3) with the results obtained from a compression test would allow the values of $\mathrm{C}_{10}$ and $\mathrm{C}_{01}$ to be estimated.

\section{ESTIMATION OF MATERIAL CONSTANTS}

Experiments were carried out on an MTS 810 testing machine (Fig. 4c) and based on the ISO 7743 standard. Cylindrical samples were made of a chloroprene rubber CR with a diameter of 29 $\mathrm{mm}$ and length of $13 \mathrm{~mm}$. Specimens were axially compressed under a strain control with the rate of $10 \mathrm{~mm} / \mathrm{min}$ till the axial strain $\varepsilon \approx-0.3$ was reached. It corresponded with the absolute change in the height by $\Delta z \approx 4 \mathrm{~mm}$. Next the strain was relieved with the same rate. Contact surfaces between the sample and testing machine plates were coated with a lubricant. This allowed friction force to be reduced during a radial expansion of the material and prevented specimen deformation into a barrel shape during compression (Fig. 4b). The described loading cycle was repeated three times for each sample. In total, there were 15 samples. The relation between the stress and strain recorded during the single experiment is presented in Fig. 5. It shows an elastic hysteresis of the tested rubber. The compression curve changes during the experiment and stabilizes after the first loading cycle. The stress value at the maximum strain is reduced in the second cycle. The relaxation time of the compressed material is quite long, because stress increases in the second and third cycles can be observed for strains larger than $\varepsilon \approx 0,04$ which means that lasting deformations took place.

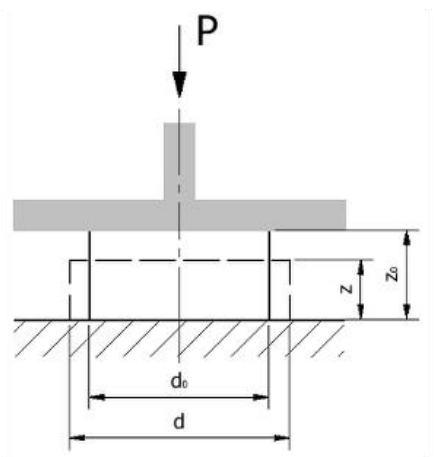

a)

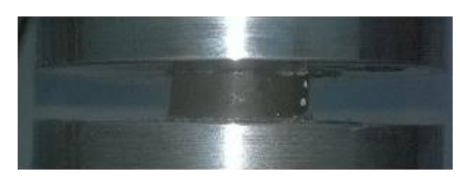

b)

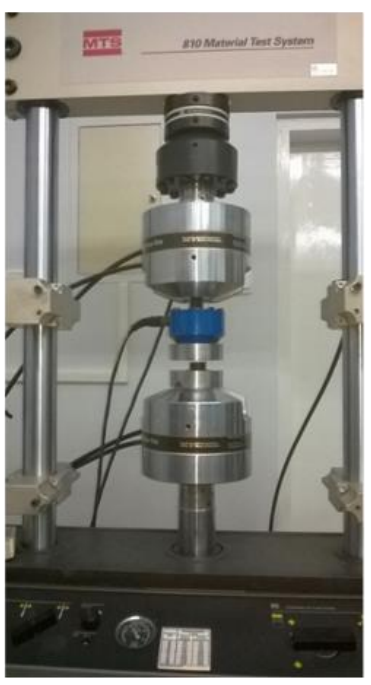

c)
Fig. 4. Experimental technique: a) specimen dimensions; b) specimen $\varnothing 29 \times 13$ during test, c) test stand

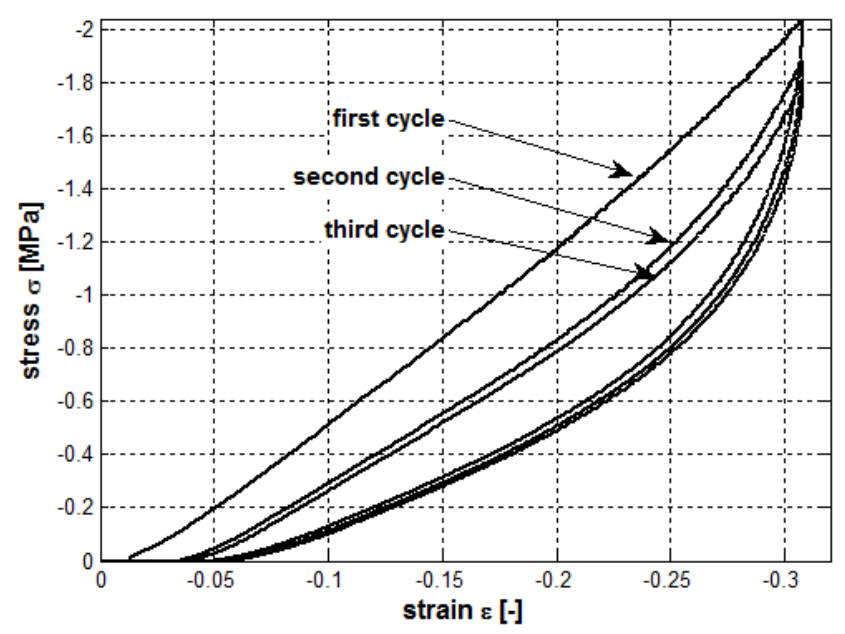

Fig. 5. An example of an elastic histeresis for a tested rubber sample

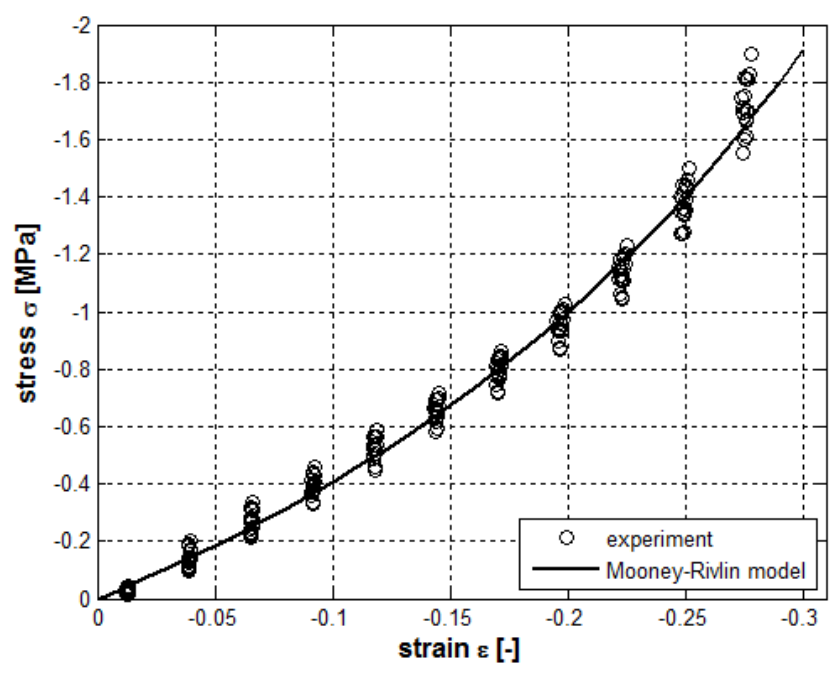

Fig. 6. Comparison between Mooney-Rivlin model curve and experimental results for the compression test 
Material constant estimations were based on the compression curve from the third loading cycle. The reason is that the last curve should be similar to a stabilised material compression response in the working spring. When the object of interest is the force needed for an initial deformation of the rubber cylinders directly during spring assembly, the curve from the first cycle should be used instead. The material constants $C_{10}$ and $C_{01}$ were determined using the least squares method by fitting function (3) to the experimental curves. For further analysis, the mean values of $\mathrm{C}_{10}$ and $\mathrm{C}_{01}$ from all 15 samples were used. Their values were equal to $C_{10}=0.2461$ and $C_{01}=0.3271$. In Fig. 6 , a theoretical curve for the Mooney-Rivlin model was compared with the experimental results. Strain values visible in fig. 6 were shifted by $\varepsilon \approx 0.04 \mathrm{com}$ pared to Fig. 5 due to the lasting deformation occurring in the third cycle.

\section{SPRING ASSEMBLY PRELOAD FORCE}

An elastic reaction force exerted by a single rubber cylinder in an unloaded Neidhart spring could be recreated by pushing a cylinder sample into the $90^{\circ} \mathrm{V}$ groove, as it is shown in Fig. 7 . Considering only one rubber element instead of all four at the same time is possible because of the two symmetry planes that exist in the examined spring. Elastic deflection of the sample is described by the variable $\Delta h=h_{0}-h$. For the compressed cylindrical sample of the dimensions $\varnothing 10 \times 42 \Delta \mathrm{h}$ is equal to ca. $3 \mathrm{~mm}$, which corresponds to the deflection in typical Neidhart rubber springs.

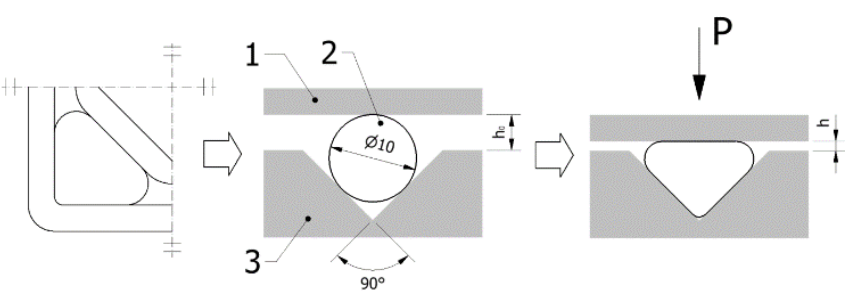

Fig. 7. Alternative setting for a quarter of Neidhart rubber spring: 1 - pressure plate, 2 - rubber cylinder, 3 - plate with groove

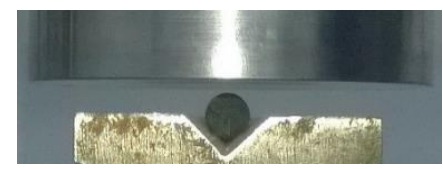

Fig. 8. Sample $\varnothing 10 \times 42$ on testing stand

The experiment was prepared and carried out to simulate the situation above. A cylindrical sample of the dimensions $\varnothing 10 \times 42$, placed horizontally in the $\mathrm{V}$ groove cut in the steel plate (Fig. 8), was compressed along its axis. The test was repeated for four samples on the same testing machine, like in the previous experiment. During the test, compression force and deflection $\Delta \mathrm{h}$ were recorded. Each sample underwent three identical loading cycles where $\Delta \mathrm{h}$ reached a maximum value of $3 \mathrm{~mm}$. For further analysis, only the compression curve $\mathrm{P}(\Delta h)$ from the last cycle, after stabilization of the sample response, was used.

\section{FEM SIMULATIONS}

Based on the previous experiment, the exact system was rec- reated in the ANSYS 16 version FEM simulation program. The simulation model (Fig. 9) consists of two rigid bodies (grooved plate and pressure plate) and a deformable body (cylindrical rubber element). Material constants for the CR rubber necessary to carry out the simulation were assumed according to the mean values $C_{10}$ and $C_{01}$ presented in the earlier section. The existence of two symmetry planes in the model - longitudinal and transverse - allows only a quarter of the whole system to be recreated. In the place of the removed fragment, the symmetry boundary conditions have to be placed on specific surfaces. Thanks to this simplification, the computing resources and time needed for performing the simulation were reduced. The model of the single rubber cylinder visible in Fig. 9 was divided into 17,000 finite elements of the type SOLID285. On the section surfaces of each of the three parts, the symmetry condition was assigned. The grooved plate was fixed in place and loading was exerted by a pressure plate with displacement applied vertically. The force necessary for deformation of the sample by the given value $(\Delta \mathrm{h}=3 \mathrm{~mm})$ was recorded as a reaction force on the pressure plate during the simulation.

A comparison of the simulation and experimental results is presented in Fig. 10. Similar progress of the compressive force as a function of $\Delta \mathrm{h}$ for FEM and experimental values allows one to assume that the prepared simulation reflects the actual physical process to a satisfactory degree. Consequently, it is justified to continue analysis for different geometrical dimensions of cylindrical samples, based further only on FEM simulations.

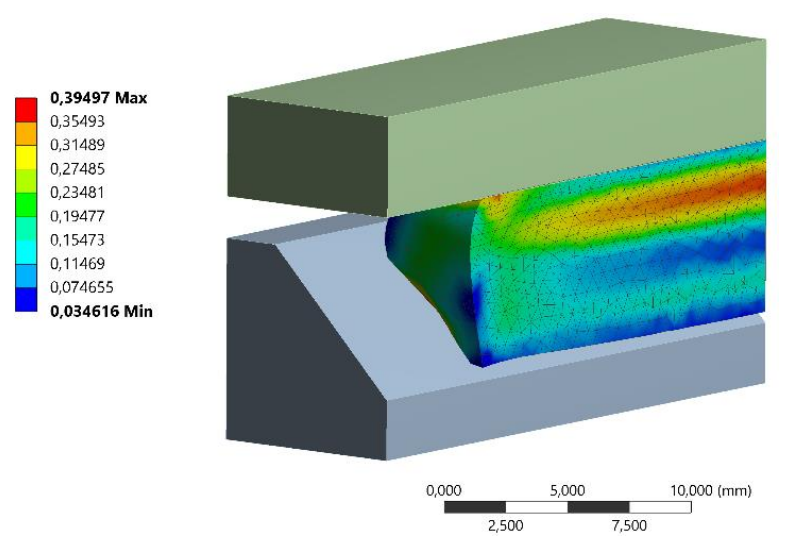

Fig. 9. FEM simulation of cylindrical sample compression. Results shown as maximum principal elastic strain of material

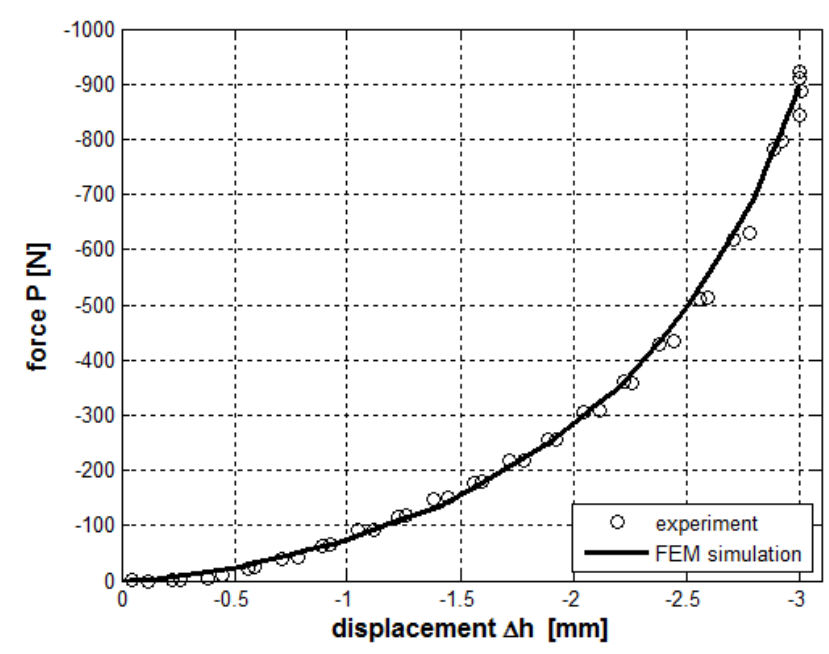

Fig. 10. Comparison of experimental results with the FEM simulation of pressing cylindrical rubber sample into groove 
The maximum amplitudes of the load for elastomeric parts are important in terms of their fatigue durability (Luo and Mortel, 2009). The maximum principal elastic strain of the sample is shown in Fig. 9. It can be used as a measure of the material equivalent load in complex loading situations (Mars and Fatemi, 2002). Analysing the distribution of the mentioned strain shows that the most loaded area is the sample interior near the contact with the pressure plate. Moreover, because of the material expansion along the groove, the face of the sample begins to bulge, which leads to the collapse of the face edge during contact between the sample and both plates. Increases in material load because of this are more significant for contact with the pressure plate.

\section{RELATION BETWEEN RUBBER CYLINDER DIMENSIONS AND ELASTIC REACTION FORCE}

The next step was an attempt to devise a functional relation between the force $P$ resulting from an initial deformation of rubber cylinders in a Neidhart spring and their dimensions - diameter $d$ and length $\mathrm{L}$. For this purpose, FEM simulations were carried out for a limited range of cylinder dimensions. It was assumed that the diameters would fit in the range from 10 to $30 \mathrm{~mm}$ and length from 40 to $80 \mathrm{~mm}$. Since the sample diameters are different compared to the ones that were tested earlier, it was necessary to define the value of $\Delta \mathrm{h}$ for other cases of diameter. It has been assumed that an elastic deflection $\Delta h$, being the result of an initial deformation of rubber cylinders, will be equal to $30 \%$ of the diameter size d:

$$
\Delta h=0.3 d
$$

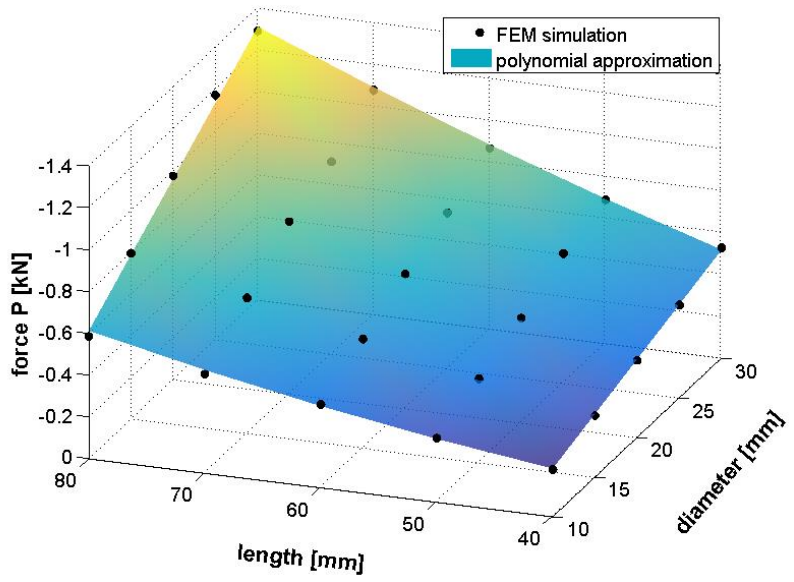

Fig. 11. FEM simulation results for compressive force $P$ as a function of rubber cylinder diameter $d$ and length $L$, and approximation of those results by the polynomial function

The proposed value corresponds to the initial deflection value in typical Neidhart springs. In total, 25 simulations were carried out for 5 values of diameter $d$ and 5 values of length $L$ from the range that was mentioned earlier. FEM simulation results are presented in the form of a 3D scatter plot in Fig. 11. It shows the values of the compressive force $P$ for the initial deflection $\Delta h=0.3 \mathrm{~d}$ for given values of rubber cylinder dimensions.

Further, the obtained FEM results were approximated. The relation describing the compressive force $\mathrm{P}$ as a function of two variables $P(d, L)$ was introduced as a polynomial function due to its versatility and ease of use in practical applications:

$P(d, L)=\sum_{i+j=0}^{i+j=N} p_{i j} d^{i} L^{j}$

where: $N=2, i=\{0,1\}, j=\{0,1,2\}$.

After expanding (4) for given values of $\mathrm{N}, \mathrm{i}$ and $\mathrm{j}$, the result is:

$P(d, L)=p_{00}+p_{10} d+p_{01} L+p_{11} d L+p_{02} L^{2}$

Following the approximation of FEM results, the polynomial coefficients presented below were obtained:

$$
\begin{aligned}
& p_{00}=-155.6 \mathrm{~N} \\
& p_{10}=6.02 \frac{\mathrm{N}}{\mathrm{mm}} \\
& p_{01}=4.8 \frac{\mathrm{N}}{\mathrm{mm}} \\
& p_{11}=-0.51 \frac{\mathrm{N}}{\mathrm{mm}^{2}} \\
& p_{02}=-0.077 \frac{\mathrm{N}}{\mathrm{mm}^{2}}
\end{aligned}
$$

The correlation coefficient for data received from the FEM simulation and equation (5) was $R^{2}=0.9967$ and root mean square error RMSE $=0.018$. Function (5) for the given input of the rubber cylinder diameter and length allows the value of compressive force to be estimated, which is also the elastic reaction force needed for an initial deformation of the rubber cylinders used in Neidhart springs. This is presented as a surface plot in Fig. 11. The developed relationship applies only to elastic elements made of chloroprene rubber $\mathrm{CR}$, whose material constants were estimated in this paper.

\section{CONCLUSIONS}

The elements to which a Neidhart spring owes its elastic properties are rubber cylinders. To analyse spring construction and operation, knowledge about the characteristics of the material used for cylinders is necessary. The conducted tests of axial compression for cylindrical rubber samples allow the material constants of the Mooney-Rivlin model to be determined, which was utilised for further work. One of the features specific for a Neidhart spring is its preload. This is the effect of initial deformation of rubber elements during spring assembly. Preload depends mainly on the geometry of rubber parts and the type of material used for their manufacture. The result of the conducted experiments and numerical simulations was the development of a functional relation which, for a given diameter and length of rubber cylinder, allows its elastic force reaction in the assembled spring to be estimated. The presented methodology is universal, because it can also be applied for cylindrical elements made of a different rubber type than that specified in this paper.

\section{REFERENCES}

1. Arruda E. M., Boyce M. C. (2000), Constitutive models of rubber elasticity: a review, Rubber Chemistry and Technology, 73(3), 504-523. 
2. Banić M. S., et al. (2012), Prediction of heat generation in rubber or rubber-metal springs, Thermal Science, 16, Suppl. 2, 527-539.

3. Baranowski P., Bogusz P., Gotowicki P., Małachowski J. (2012), Assessment of mechanical properties of off-road vehicle tire: coupons testing and FE model development, Acta Mechnica et Automatica, 6(2), 17-22.

4. Bower A. F. (2010), Applied mechanics of solids, CRC Press, Boca Raton.

5. Chokanandsombat Y., Sirisinha C. (2013), MgO and ZnO as reinforcing fillers in cured polychloroprene rubber, Journal of Applied Polymer Science, 128(4), 2533-2540.

6. Gent A. N., Suh J. B., Kelly S. G. (2007), Mechanics of rubber shear springs, International Journal of Non-Linear Mechanics, 42(4), $241-249$.

7. Hassan M. A., Abouel-Kasem A., Mahmoud A. El-Sharief, Yusof F. (2012), Evaluation of the material constants of nitrile butadiene rubbers (NBRs) with different carbon black loading (CB): FEsimulation and experimental, Polymer, 53(17), 3807-3814.

8. ISO 7743 (2011), Rubber, vulcanized or thermoplastic Determination of compression stress-strain properties.

9. Kim B., Lee S. B., Lee J., Cho S., Park H., Yeom S., Park S. H. (2012), A comparison among Neo-Hookean model, Mooney-Rivlin model and Ogden model for chloroprene rubber, International Journal of Precision Engineering and Manufacturing, 13(5), 759-764.

10. Lee B. S., Rivin E. I. (1996), Finite element analysis of loaddeflection and creep characteristics of compressed rubber components for vibration control devices, ASME Journal of Mechanical Design, 118, 328-336.

11. Lu Y.T., Zhu H. X., Richmond S., Middleton J. (2010), A viscohyperelastic model for skeletal muscle tissue under high strain rates, Journal of Biomechanics, 43(13), 2629-2632.

12. Luo R. K., Mortel W. J., Wu X. P. (2009), Fatigue failure investigation on anti-vibration springs, Engineering Failure Analysis, 16(5), 1366-1378.
13. Mars W. V., Fatemi A. (2002), A literature survey on fatigue analysis approaches for rubber, International Journal of Fatigue, 24(9), 949-961.

14. Mooney M. (1940), A theory of large elastic deformation, Journal of Applied Physics, 11(9), 582-592.

15. Neidhart H. (1951), Elastic joints, US patent 2712742.

16. Neidhart R. (1969), Special spring unit, Rubbers Handbook, MorganGrampian, London

17. Paluch M. (2006), Fundamentals of the Theory of Elasticity and Plasticity, Wydawnictwo Politechniki Krakowskiej, Kraków (in Polish).

18. Rivin E. I. (2003), Passive vibration isolation, ASME Press, New York.

19. Rivin E. I., Lee B. S. (1994), Experimental study of load-deflection and creep characteristics of compressed rubber components for vibration control devices, ASME Journal of Mechanical Design, 116, 539-549.

20. Rivlin R. S. (1948), Large elastic deformations of isotropic materials. IV. Further developments of the general theory, Philosophical Transactions of the Royal Society of London. Series A, Mathematical and Physical Sciences, 241(835), 379-397.

21. Samaca Martinez J.R., Le Cam J.-B., Balandraud X., Toussaint E., Caillard J. (2013), Filler effects on the thermomechanical response of stretched rubbers, Polymer Testing, 32(5), 835-841.

22. Wodziński P. (2003), Application of elastic rubber suspensions in vibrating screens and feeders (in Polish), Inżynieria Mineralna, 3, 109-114.

23. http://www.rosta.ch

Acknowledgements: This research was supported in part by PL-Grid Infrastructure. 